\title{
Percutaneous Ultrasound-Guided Superior and Inferior Mesenteric Vein Access for Portal Vein Recanalization- Transjugular Intrahepatic Portosystemic Shunt: A Case Series
}

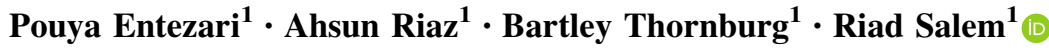

Received: 3 September 2020 / Accepted: 6 November 2020/Published online: 23 November 2020

(C) Springer Science+Business Media, LLC, part of Springer Nature and the Cardiovascular and Interventional Radiological Society of Europe (CIRSE) 2020

\begin{abstract}
Objective To describe the technique and outcomes of mesenteric access under ultrasound guidance to perform portal vein recanalization-transjugular intrahepatic portosystemic shunt (PVR-TIPS).

Methods Four patients (3 male: 1 female, mean age: 46.2 years; range 38-64 years) with portal vein thrombosis (PVT) and cavernous transformation were eligible for PVR-TIPS. Due to inaccessible splenic vein (one patient with history of splenectomy and 3 patients with unavailable splenic vein during the procedure), noninvasive direct puncture of superior $(n=3)$ and inferior $(n=1)$ mesenteric vein was conducted under ultrasound guidance to obtain access for PVR-TIPS.

Results Trans-mesenteric access and PVR-TIPS were successful in all patients at first attempt. No immediate complication was observed following the procedures. Follow-up imaging with computed tomography (CT) scan and Doppler ultrasound revealed patent TIPS and portal venous vasculature in all patients.

Conclusion Percutaneous noninvasive transmesenteric access is a feasible approach for PVR-TIPS in patients with inaccessible splenic veins.
\end{abstract}

Electronic supplementary material The online version of this article (https://doi.org/10.1007/s00270-020-02713-0) contains supplementary material, which is available to authorized users.

Riad Salem

r-salem@northwestern.edu

1 Department of Radiology, Section of Interventional Radiology, Northwestern Memorial Hospital, 676 N. St. Clair, Suite 800, Chicago, Illinois 60611, USA
Level of evidence IV This journal requires that authors assign a level of evidence to each article. For a full description of these Evidence-Based Medicine ratings, please refer to the Table of Contents or the online Instructions to Authors www.springer.com/00266.

Keywords Portal vein thrombosis · Portal vein recanalization-transjugular intrahepatic portosystemic shunt $\cdot$ Splenic vein $\cdot$ Mesenteric access

\section{Introduction}

Portal vein thrombosis (PVT) is reported in both cirrhotic and non-cirrhotic patients [1]. Besides aggravating portal hypertension sequelae, PVT increases postoperative complications during liver transplantation (LT), and hence, PVT is considered as a relative contraindication to LT [2]. Portal vein recanalization-transjugular intrahepatic portosystemic shunt (PVR-TIPS) re-establishes PV flow, relieves portal hypertension symptoms, and improves patients' eligibility for LT [3]. Over time, the method has evolved to the trans-splenic approach with a high rate of success [1, 4]. We report our experience of performing PVR-TIPS by percutaneous puncture of the superior/inferior mesenteric veins (SMV/IMV) in patients with thrombosed splenic veins (SV). 


\section{Materials and Methods}

Patients with chronic PVT eligible for PVR-TIPS and inaccessible for trans-splenic approach underwent treatment using direct, ultrasound-guided SMV/IMV punctures.

\section{Procedures and Technical Aspects}

In all patients, a 10-Fr sheath (Cook, Bloomington, Indiana) was advanced into the right hepatic vein via the right internal jugular vein (IJV). Primary transhepatic and transsplenic approaches failed in all patients. Subsequently, using ultrasound guidance, a 21-gauge needle (Cook, Bloomington, Indiana) was advanced transabdominally into the SMV (Fig. 1) in 3 patients and IMV in one patient. The SMV was thrombosed in 1 of the 3 patients and in the patient where IMV puncture was performed.

In patients \#1 and \#3, a 4-Fr glide catheter (Terumo Medical Corp, Somerset, New Jersey) was advanced into the right PV through the SMV access. A 10-mm snare (ev3 Endovascular, Plymouth, Minnesota) was placed in the right PV as a fluoroscopic target for TIPS needle puncture. After advancing a Colapinto needle (Cook, Bloomington, Indiana) into the right $\mathrm{PV}$ and into the snare, a stiff glide (Terumo Medical Corp) wire was advanced through the needle and then captured by the snare and pulled through the percutaneous SMV skin entry site, establishing Archimedean (through and through) access. The sheath was advanced into the portal system. Portomesenteric venography was performed confirming thrombosis. After deploying a Viatorr stent graft (W.L Gore \& Associates, Flagstaff, Arizona) and dilating with a $10-\mathrm{mm}$ balloon,

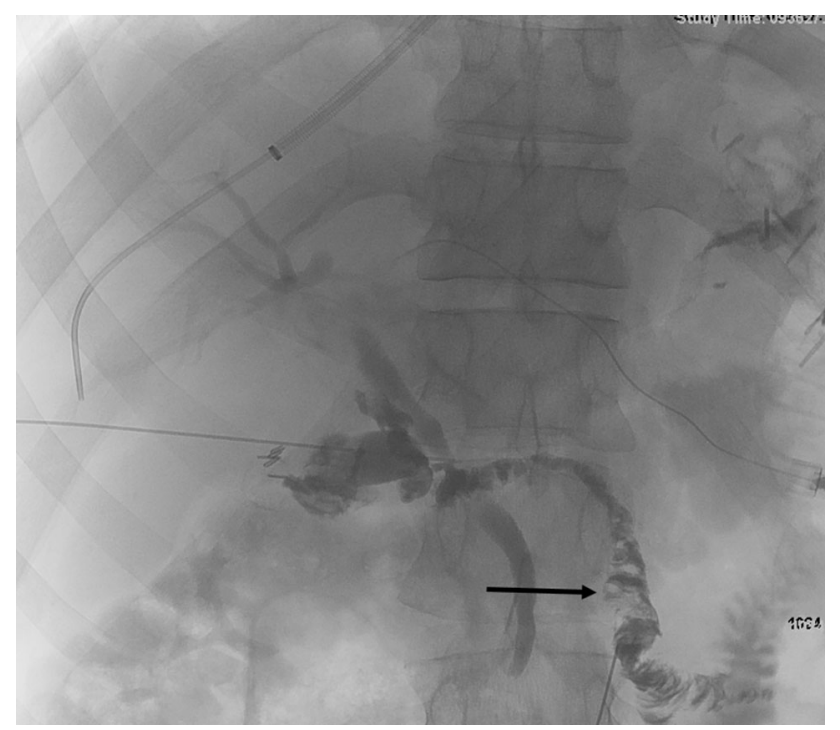

Fig. 1 Percutaneous access of SMV (arrow) was obtained to access the PV portomesenteric venography demonstrated patency and residual clot. Subsequently, pharmacomechanical thrombectomy of the MPV and SMV using Angiojet (Boston Scientific, Marlborough, Massachusetts) was performed. Subsequent stent extension and venography demonstrated patent TIPS and portal vasculature with strong flow (Fig. 2, Online Resources 1-4 for patient \#1, Online Resources 16-21 for patient \#3).

In patient \#2, the SMV access was upsized for a 4-Fr glide catheter. The catheter was advanced into a peripheral SV parenchymal branch to provide splenic access in anticipation of portal recanalization. After advancing a Gooseneck snare through the catheter and passing a 21-gauge needle through the snare, a Cope mandril wire was grasped and trans-splenic approach was achieved. A 5-Fr catheter and glidewire were advanced into the right PV using recanalization. Through both IJ and splenic accesses, snares were positioned into the right hepatic and portal veins. Using gun-sight technique [5], an 18-gauge needle was advanced through the snares, and then TIPS access was obtained. PVR-TIPS was then completed per routine methodology. Portosystemic gradient decreased to $3 \mathrm{mmHg}$. (Online Resource 5-15).

In patient \#4, the IMV was accessed under ultrasound guidance (Fig. 3). After fluoroscopic confirmation of IMV access, a 4-Fr glide wire was advanced though the thrombosed mesenteric system into the right PV. Gooseneck snare was placed into right PV to establish Archimedean access and TIPS stent was deployed as described previously. (Online Resources 22, 23).

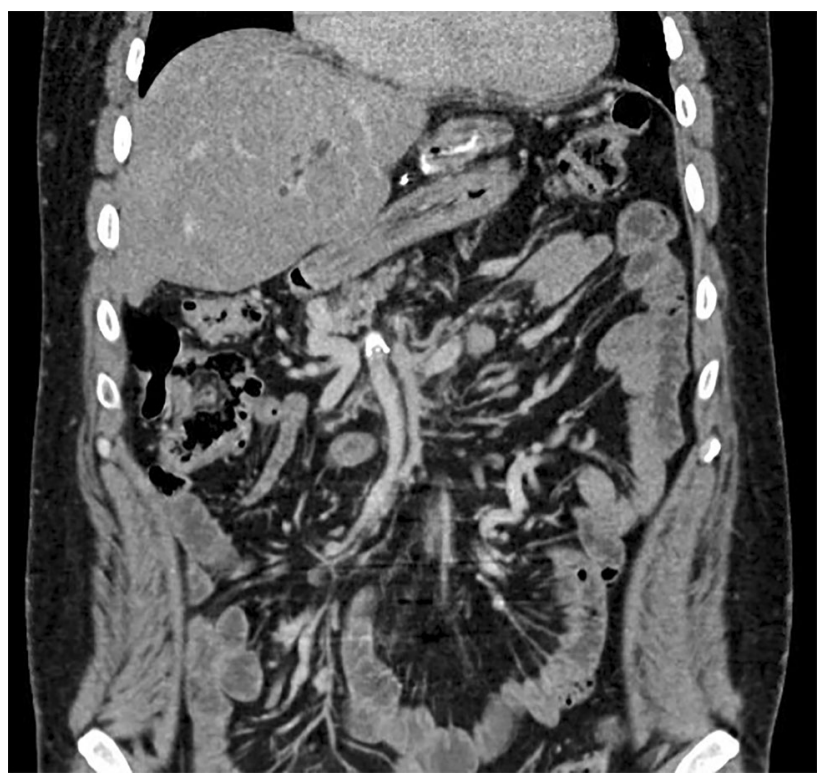

Fig. 2 Post-TIPS CT scan at 2-month follow up showing patent PV and SMV 


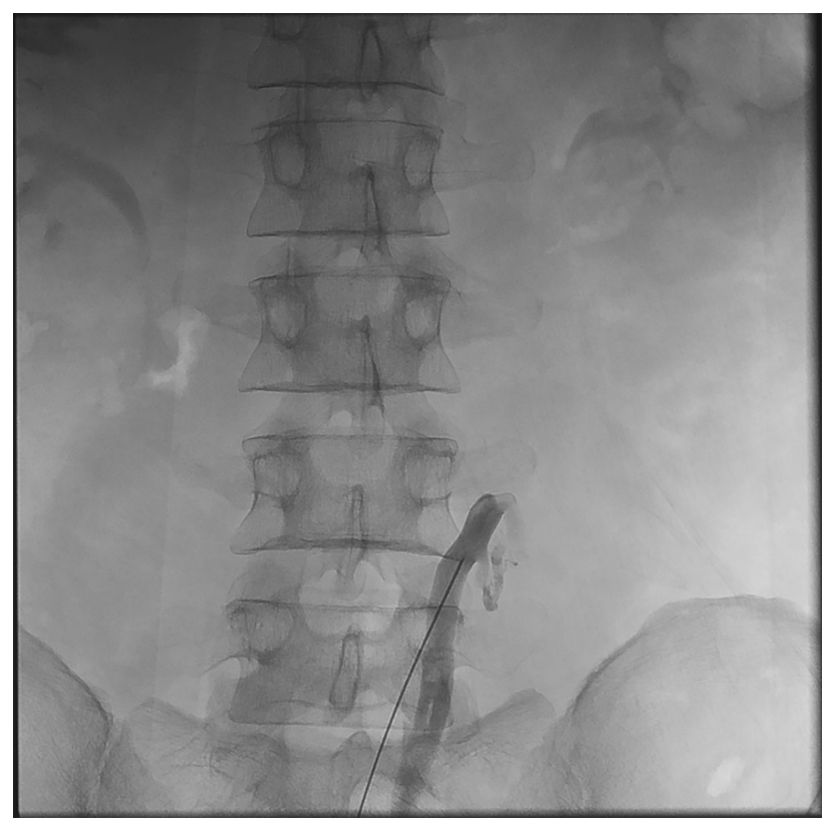

Fig. 3 Percutaneous direct puncture of IMV was obtained to access PV

\section{Results}

Four patients were treated from March to August 2020. Baseline characteristics of patients, underlying conditions, and pre-procedure findings are outlined in Table 1. There were no immediate complications, including bleeding. One-month post-procedure follow-up imaging with computed tomography (CT)-venogram and Doppler ultrasound showed widely patent PV, SV and TIPS shunt with excellent flow in all patients, and none of the patients developed any complication during this time (including delayed bleeding). Unfortunately, patient \#2 did not progress to transplant as he succumbed to Corona virus (COVID-19) infection 3 months after the procedure.

\section{Discussion}

PVT treatment is challenging and management is decided on a case-by-case basis. Anticoagulation has been proposed in cirrhotic or non-cirrhotic patients who are predisposed to venous thrombosis $[6,7]$. However, higher tendency for bleeding in cirrhotic patients and development of esophageal varices in many cases of chronic PVT render anticoagulation therapy challenging.

PVR-TIPS is effective in PVT treatment in both cirrhotic and non-cirrhotic patients $[1,4,8]$. The trans-splenic approach has become the technique of choice in PVRTIPS. In an evaluation on trans-hepatic approach, 53\% of TIPS cases failed at placement or required stent revision after first placement [9]. However, trans-splenic approach has offered a favorable technical success rate of almost $100 \%[1,4,8]$. Obtaining SV access is the main contributor to success of trans-splenic approach. Despite an acceptable safety profile, complications can occur (e.g. TIPS stenosis) [4].

In asplenic patients and patients with difficult SV access, or in patients with complete mesenteric venous thrombosis, both trans-hepatic and trans-splenic approaches face major technical challenges. There are few reports of utilizing trans-mesenteric approach for TIPS in asplenic patients with PVT [10, 11]. In these cases, a mini-laparatomy incision was required to obtain access to a peripheral mesenteric vein. We believe noninvasive, ultrasound-guided, mesenteric puncture to gain access for PVR-TIPS can be beneficial in treatment of chronic PVT in patients with unavailable SV, as demonstrated in the case

Table 1 Baseline characteristics

\begin{tabular}{lllll}
\hline $\begin{array}{l}\text { Patient } \\
\text { number }\end{array}$ & Age & Gender & Underlying condition & Baseline imaging findings \\
\hline 1 & 43 & Male & $\begin{array}{c}\text { Previous splenectomy with new-onset post-prandial pain. Initial } \\
\text { attempt of catheter-directed thrombolysis failed } \\
\text { HCC and chronic PVT in anticipation for LT }\end{array}$ & $\begin{array}{c}\text { MPV and SMV thrombosis with cavernous } \\
\text { transformation } \\
\text { Cavernous transformation of PV, cirrhosis, } \\
\text { splenomegaly, esophageal varices }\end{array}$ \\
3 & 64 & Male & Female & $\begin{array}{c}\text { Chronic PVT due to JAK2 mutation with history of } \\
\text { unsuccessful trans-splenic PVR-TIPS } \\
\text { Occluded SV, extensive thrombus in the SMV, } \\
\text { cavernous transformation of main, right, and left } \\
\text { PVs }\end{array}$ \\
4 & 40 & Male & $\begin{array}{c}\text { PVT in the setting of JAK2 and factor V Leiden mutations with } \\
\text { worsening post-prandial and extensive gastroesophageal } \\
\text { varices }\end{array}$ & $\begin{array}{c}\text { Main PV, SV, SMV could not be identified due to } \\
\text { extensive thrombosis. Only IMV could be } \\
\text { visualized }\end{array}$ \\
\hline
\end{tabular}

$M P V$, main portal vein; $S M V$, superior mesenteric vein; $H C C$, hepatocellular carcinoma; $P V T$, portal vein thrombosis; $L T$, liver transplantation; $J A K$ 2, Janus Kinase 2; PVR-TIPS, portal vein recanalization-transjugular intrahepatic portosystemic shunt; $S V$, splenic vein; $I M V$, inferior mesenteric vein 
report [12]. Further studies can evaluate long-term safety and clinical outcome of this novel procedure.

\section{Conclusion}

Percutaneous trans-mesenteric approach is a feasible alternate access route to directly perform PVR-TIPS with a favorable clinical success and safety.

Funding This study was not supported by any funding.

\section{Compliance with Ethical Standards}

Conflict of interest The authors declare that they have no conflict of interest.

Consent for Application Consent for publication was obtained for every individual person's data included in the study.

Ethical Approval All procedures performed in studies involving human participants were in accordance with the ethical standards of the institutional and/or national research committee and with the 1964 Helsinki Declaration and its later amendments or comparable ethical standards. Institutional IRB Approval for this study was obtained.

Informed Consent Informed consent was obtained from all individual participants included in the study.

\section{References}

1. Kallini JR, Gabr A, Kulik L, Ganger D, Lewandowski R, Thornburg B, et al. Noncirrhotic complete obliterative portal vein thrombosis: novel management using trans-splenic transjugular intrahepatic portosystemic shunt with portal vein recanalization. Hepatology. 2016;63(4):1387-90.

2. Yerdel MA, Gunson B, Mirza D, Karayalçin K, Olliff S, Buckels $\mathrm{J}$, et al. Portal vein thrombosis in adults undergoing liver transplantation: risk factors, screening, management, and outcome. Transplantation. 2000;69(9):1873-81.
3. Salem R, Vouche M, Baker T, Herrero JI, Caicedo JC, Fryer J, et al. Pretransplant portal vein recanalization-transjugular intrahepatic portosystemic shunt in patients with complete obliterative portal vein thrombosis. Transplantation. 2015;99(11):2347-55

4. Habib A, Desai K, Hickey R, Thornburg B, Vouche M, Vogelzang RL, et al. Portal vein recanalization-transjugular intrahepatic portosystemic shunt using the transsplenic approach to achieve transplant candidacy in patients with chronic portal vein thrombosis. J Vasc Interv Radiol. 2015;26(4):499-506.

5. Haskal ZJ, Duszak R Jr, Furth EE. Transjugular intrahepatic transcaval porto-systemic shunt: the gun-sight approach. J Vasc Interv Radiol. 1996;7(1):139-42.

6. EASL Clinical Practice Guidelines. Vascular diseases of the liver. J Hepatol. 2016;64(1):179-202.

7. DeLeve LD, Valla DC, Garcia-Tsao G. Vascular disorders of the liver. Hepatology. 2009;49(5):1729-64.

8. Thornburg B, Desai K, Hickey R, Kulik L, Ganger D, Baker T, et al. Portal vein recanalization and transjugular intrahepatic portosystemic shunt creation for chronic portal vein thrombosis: technical considerations. Tech Vasc Interv Radiol. 2016;19(1):52-60.

9. Senzolo M, Tibbals J, Cholongitas E, Triantos C, Burroughs A, Patch D. Transjugular intrahepatic portosystemic shunt for portal vein thrombosis with and without cavernous transformation. Aliment Pharmacol Ther. 2006;23(6):767-75.

10. Matsui O, Yoshikawa J, Kadoya M, Gabata T, Takashima T, Urabe $\mathrm{T}$, et al. Transjugular intrahepatic portosystemic shunt after previous recanalization of a chronically thrombosed portal vein via a transmesenteric approach. Cardiovasc Intervent Radiol. 1996;19(5):352-5.

11. Sabri SS, Caldwell SH, Kumer SC, Schmitt TM, Maluf DG, Angle JF, et al. Combined transmesenteric and transhepatic recanalization of chronic portal and mesenteric vein occlusion to treat bleeding duodenal varices. J Vasc Interv Radiol. 2014;25(8):1295-9.

12. Farsad K, Zaman A. Percutaneous Transmesenteric Portal Vein Recanalization and Transjugular Direct Intrahepatic Portosystemic Shunt Creation for Chronic Portal Vein Occlusion. J Vasc Interv Radiol. 2019;30(6):892-3.

Publisher's Note Springer Nature remains neutral with regard to jurisdictional claims in published maps and institutional affiliations. 